\title{
The Subthreshold Relation between Cortical Local Field Potential and Neuronal Firing Unveiled by Intracellular Recordings in Awake Rats
}

\author{
Michael Okun, Amir Naim, and Ilan Lampl \\ Department of Neurobiology, Weizmann Institute of Science, Rehovot 76100, Israel
}

In most of the in vivo electrophysiological studies of cortical processing, which are extracellular, the spike-triggered local field potential average (LFP STA) is the measure used to estimate the correlation between the synaptic inputs of individual neuron and the local population. To understand how the magnitude and shape of LFP STA reflect the underlying correlation of synaptic activities, the membrane potential of the firing neuron has to be recorded together with the LFP. Using intracellular recordings from the cortex of awake rats, we found that for a large range of firing rates and for different behavioral states, the LFP STA represents both in its waveform and its magnitude the cross-correlation between the membrane potential of the neuron and the LFP. This data, supported by further analysis, suggests that LFP STA does not represent large network events specific to the spike times, but rather the synchrony between the mean synaptic activity of the population and the membrane potential of the single neuron, present both around spike times and in the intervals between spikes. Furthermore, it introduces a novel interpretation of the available data from unit and LFP extracellular recording experiments.

\section{Introduction}

Extracellular recordings constitute the most widespread technique of electrophysiology in vivo. In the cortex, an extracellular electrode picks up two distinct classes of neuronal signals, produced by current flow across cell membranes in a local volume of a few hundreds of micrometers around the electrode tip. The high frequency $(>0.5 \mathrm{kHz})$ signal mainly reflects spiking of neurons near the electrode tip and is used to record single or multiunit activity. The signal in frequencies below $\sim 200 \mathrm{~Hz}$, termed the local field potential (LFP), is mostly due to synaptic activity (Mitzdorf, 1985; Buzsaki, 2006; Rasch et al., 2008; Katzner et al., 2009). Recently, cortical LFP recordings are gaining a renewed popularity, as it becomes clear that LFP signals contain precise neuronal correlates of a whole range of behaviors, in some cases even more so than spikes (Pesaran, 2009). Furthermore, LFP is extremely important for the interpretation of functional magnetic resonance imaging blood oxygenation leveldependent data (Niessing et al., 2005; Viswanathan and Freeman, 2007; Logothetis, 2008). From the experimental point of view, LFP signals are easy to obtain, as one actually has to filter out this signal to obtain unit recordings.

For many questions having to do with cortical processing, it is imperative to examine the relationship between spikes and LFP rather than considering just the spikes or just the LFP (Gray

\footnotetext{
Received 0ct. 11, 2009; revised Jan. 30, 2010; accepted Feb. 15, 2010.

This work was supported by grants from United States-Israel Binational Science Foundation (2007444) and Israel Science Foundation (326/07). We thank Amos Arieli, Omri Barak, David Ferster, and Rony Paz for their comments on this manuscript, and all the members of the Lampl laboratory for their helpful contribution to this work.

Correspondence should be addressed to either Michael Okun or llan Lampl, Department of Neurobiology, Weizmann Institute of Science, Rehovot 76100, Israel, E-mail: michael.okun@mail.huji.ac.il or ilan.lampl@weizmann.ac.il.

D01:10.1523/JNEUROSCI.5062-09.2010

Copyright $\odot 2010$ the authors $\quad 0270-6474 / 10 / 304440-09 \$ 15.00 / 0$
}

and Singer, 1989; Eggermont and Smith, 1995; Steriade et al., 1996; Destexhe et al., 1999; Pesaran et al., 2002; Mehring et al., 2003; Henrie and Shapley, 2005; Rasch et al., 2008; Ray et al., 2008; Tiesinga et al., 2008; Xing et al., 2009). The typical measure used to correlate the activity of individual neurons to the LFP signal is the spike-triggered average (STA) of the field potential (Gray and Singer, 1989; Eggermont and Smith, 1995; Destexhe et al., 1999; Fries et al., 2001; Pesaran et al., 2002; Goldberg et al., 2004; Ray et al., 2008; Katzner et al., 2009; Nauhaus et al., 2009). In vivo, synaptic inputs to the cortical neurons are the primary cause for their membrane potential $\left(V_{\mathrm{m}}\right)$ fluctuations, responsible for spiking (Steriade, 2001), which is in particular evident from the synchrony of simultaneously recorded nearby neurons (Lampl et al., 1999; Hasenstaub et al., 2005; Volgushev et al., 2006; Okun and Lampl, 2008; Poulet and Petersen, 2008). Therefore, both the waveform and the magnitude of the LFP STA are mainly determined by the relation between the LFP signal and neuron's membrane potential. Indeed, LFP in the vicinity of a cortical neuron is known to be correlated with its $V_{\mathrm{m}}$ (Deweese and Zador, 2004; Haider et al., 2006; Rudolph et al., 2007; Poulet and Petersen, 2008). However, it remains unclear how the $V_{\mathrm{m}}$-LFP crosscorrelation (VmLFPcc) is reflected in the relationship between the spikes of the cell and the LFP. We therefore set out to investigate the mutual relationships between $V_{\mathrm{m}}$, spikes and LFP, by means of simultaneous intracellular and nearby LFP recordings in the cortex of awake rats.

\section{Materials and Methods}

Experimental procedures. All surgical and experimental procedures were in accordance with the regulations of the Weizmann Institute Animal Care and Use Committee. In the experiments we used Wistar rats, 4-5 weeks old. For the initial surgery, the animals were anesthetized with 
isoflurane (2-3\%) and placed in a standard stereotaxic device with blunt ear bars. Body temperature was kept at $37.5 \pm 0.5^{\circ} \mathrm{C}$ using a heating blanket and a rectal thermometer (TC-1000, CWE). During the surgery, a part of the skull was exposed, then a pair of bolts for head fixation and a small plastic chamber were attached to the skull, either by cyanoacrylate glue, acrylic cement and miniature screws or by glass ionomer luting and acrylic cements. After the surgery, antibiotics (Pen-strep, Eurovet; $2 \mathrm{ml} /$ $\mathrm{kg}$, i.m.) and analgesics (Rimadyl, Pfizer; $5 \mathrm{mg} / \mathrm{kg}$, s.c.) were administered. Following recovery, the animals underwent 5-7 training sessions, spread across 3-5 consecutive days. During the training, they were accustomed to sitting quietly with their head fixed inside a plastic tube with an opening ( $\sim 5 \mathrm{~cm}$ in diameter) in its top part, which allowed accessing the head fixation bolts and the recording chamber. The duration of the head-fixation session was gradually increased, reaching $30 \mathrm{~min}$ and above at the end of the training. Some of the animals were water deprived for $\sim 12 \mathrm{~h}$ before the training sessions, and mango juice was made available to them while they stayed inside the tube. According to our experience, such a positive reinforcement helps to accustom the rats to sitting inside the tube in the experimental setup. In addition, the wider behavior repertoire allows to discriminate between different states of the animal.

Recording sessions started once the training stage was completed. The rats were anesthetized with isoflurane for a short period of $\sim 15-20 \mathrm{~min}$, during which a small craniectomy in the skull (inside the recording chamber) and a tiny incision in the dura for the insertion of the electrodes were performed. Following the procedure the animals were released to a cage. Every several minutes a drop of Ringer solution was put over the craniectomy to prevent the exposed brain from drying up. After $\sim 1 \mathrm{~h}$, which ensured complete recovery from the brief anesthesia, as evidenced by a fully normal behavior of the animal, it was head-fixed within the plastic tube to which it was accustomed during the training. Every rat participated in 1-3 recording sessions of 30-80 min, each conducted on a different day.

Intracellular recordings were performed using the standard "blind" technique. We have used both patch and sharp electrodes, pulled from borosilicate micropipettes (outer and inner diameters: 1.5 and $0.86 \mathrm{~mm}$, respectively; Sutter Instruments), with a P-97 micropipette puller (Sutter Instruments). The sharp pipettes were filled with $2 \mathrm{~m} \mathrm{~K}$-acetate and had a resistance of $40-100 \mathrm{M} \Omega$. The patch solution contained the following (in $\mathrm{mm}$ ): $136 \mathrm{~K}$-gluconate, $10 \mathrm{KCl}, 5 \mathrm{NaCl}, 10 \mathrm{HEPES}, 1 \mathrm{MgATP}, 0.3 \mathrm{NaGTP}$, and 10 phosphocreatine $(310 \mathrm{mOsm})$, and had a resistance of 4-8 $\mathrm{M} \Omega$. For the LFP recordings we have used parylene insulated, tungsten electrodes, having $0.5-1 \mathrm{M} \Omega$ impedance at $1 \mathrm{kHz}$ (A-M Systems). The tungsten electrode was inserted a few hundred micrometers from the entrance point of the intracellular pipette and placed at a depth of 700-900 $\mu \mathrm{m}$ below the pia. Based on the penetration angles and the depths of the recordings we estimate that the distance between recording sites was $300-700 \mu \mathrm{m}$.

The intracellular signals were amplified using Axoclamp-2B or Axoclamp-900A amplifiers (Molecular Devices). The LFP signals were amplified with an MCP-plus-8 amplifier (Alpha Omega Engineering). Both signals were digitized at $10 \mathrm{kHz}$ and streamed to a computer disk using a custom data acquisition software system written in LabVIEW 8.5 (National Instruments). We used a $25 \mathrm{fps}$ video camera (SDC-415, Samsung) to monitor the animal behavior and also saved the video-stream for offline analysis (see the supplemental Movies, available at www. jneurosci.org as supplemental material).

In addition to the awake recordings we have performed several experiments in anesthetized rats. In these recordings the rats were anesthetized with ketamine and xylazine followed by halothane, as previously described (Katz et al., 2006; Heiss et al., 2008). The data from anesthetized animals is not included in the analysis, unless explicitly stated otherwise.

Data analysis. The analysis of the recordings was performed using custom software written in MATLAB 7.8.0 (The MathWorks). For the data analysis, the $V_{\mathrm{m}}$ traces were offline digitally high-pass filtered above $1 / 3 \mathrm{~Hz}$, to remove slow drifts. Spikes were detected by a derivative-based threshold, and then removed by spline interpolation based on 5 samples on each side of the action potential. The spike times were stored for the computation of the spike-triggered averages.
Cross-correlations of signal pairs were computed for entire recording intervals at once, after the signals were down-sampled to $1 \mathrm{kHz}$, according to the following formula:

$$
C C(\tau)=\frac{\sum_{t=1}^{n} x(t) y(t+\tau)}{\sqrt{\sum_{t=1}^{n} x^{2}(t)} \sqrt{\sum_{t=1}^{n} y^{2}(t+\tau)}},
$$

where $x(t)$ and $y(t)$ are two mean subtracted time series. To compute the $V_{\mathrm{m}}$-LFP cross-correlation without taking into account intervals around spikes (see Fig. 5), the above expression was used with the summation done only over time points that are at least 20,40 , or $100 \mathrm{~ms}$ away from any spike. The cross-correlations were also computed by dividing each recording into $2 \mathrm{~s}$ intervals, calculating the above expression separately for each interval, and then averaging the result. The two methods provide essentially the same results (data not shown), but the latter also allowed to compute confidence intervals, which in particular were used to determine whether VmLFPcc is significant.

The LFP STA was estimated by computing the cross-covariance (i.e., the numerator in the formula above) of the spike train, represented using 1 millisecond bins, and the LFP. The result was divided by the number of spikes in the spike train. Normalized LFP STA was computed by dividing the LFP STA by the SD of the LFP signal. For the visual comparison of the waveforms of LFP STA and VmLFPcc (as in Figs. 3, 6 , below), the STA was scaled to have the same (Euclidian) norm as the VmLFPcc vector. The similarity score between the two waveforms is simply the correlation of the two vectors in a $1 / 2 \mathrm{~s}$ interval around the 0 time lag, shifted with respect to each other ( $\pm 25 \mathrm{~ms}$ at most) to achieve optimal alignment.

The coherence estimates between $V_{\mathrm{m}}$ and LFP or between the spike train and LFP signals were computed using the open-source Chronux (http://chronux.org/) library for Matlab (Mitra and Bokil, 2008). Unless explicitly stated otherwise, we have used windows of $1.25 \mathrm{~s}$ and 9 tapers. We have examined the coherence in low $(0-25 \mathrm{~Hz})$ and gamma $(25-70 \mathrm{~Hz})$ frequency bands. A $V_{\mathrm{m}}$-LFP or spike-LFP coherence was considered to be significant within a band if the $95 \%$ confidence limit for at least half of the frequencies within the band was $>0$. In these cases, the average coherence across the frequencies of the $0-25 \mathrm{~Hz}$ $(25-70 \mathrm{~Hz})$ band was taken as the measure of coherence in the low (gamma) frequencies.

Cortical activity state transitions (see Figs. 8,9 ) were detected by inspecting the video recordings (see supplemental Movies 1 and 2, available at www.jneurosci.org as supplemental material). Every transition from a slower to a faster activity had to be accompanied by behavioral change, such as initiation of juice licking or grooming, or assuming a different posture. Every such change was accompanied by a decrease of at least $15 \%$ in the LFP magnitude, quantified by its SD over an interval of $20 \mathrm{~s}$. In several neurons we recorded more than one state transition (e.g., from slower activity during quiet behavior to faster activity during licking and then again a slower quiet mode), in which case a comparison between several states could be made. For neurons recorded in several behavioral states, the population analysis was based only on the interval before the first state transition.

\section{Results}

$V_{\mathrm{m}}$ and LFP in the cortex of awake rats are correlated across a rich repertoire of neuronal activities

We performed simultaneous intracellular and nearby LFP recordings in the primary somatosensory cortex $(\mathrm{S} 1)$ and the prefrontal cortex (PFC) of awake, head-fixed rats, which occasionally exhibited specific behaviors. A total of 32 neurons in S1 were recorded, of these 8 were recorded using patch pipettes and the rest with sharp pipettes. In addition, 9 cells from the PFC were recorded, 3 with patch pipettes and the rest with sharp pipettes. The cells were recorded from all the layers. Within the analyzed database we found no significant correlations between the laminar position of the neuron and the membrane potential, spiking or LFP parameters investigated in the present work. 
A

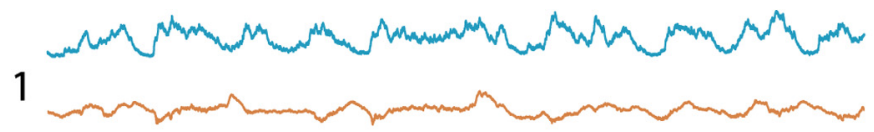

2

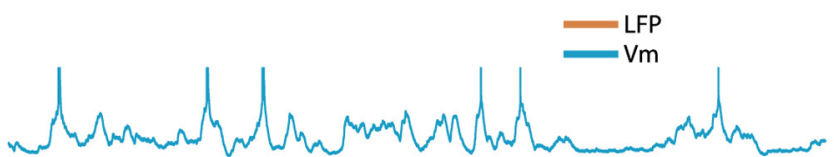

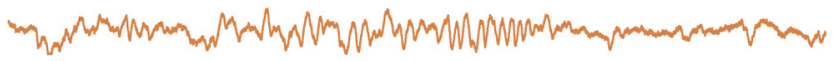

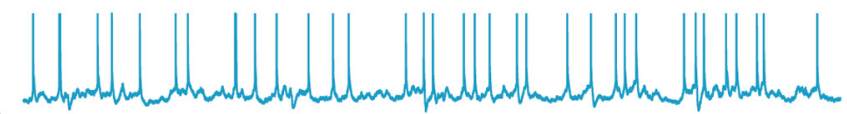

3

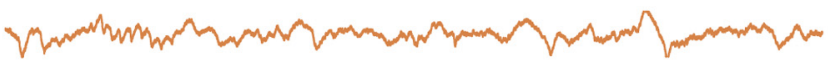

B

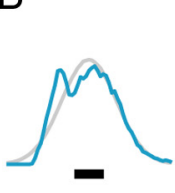

Vm distribution $\sigma=\sigma(\mathrm{Vm})$
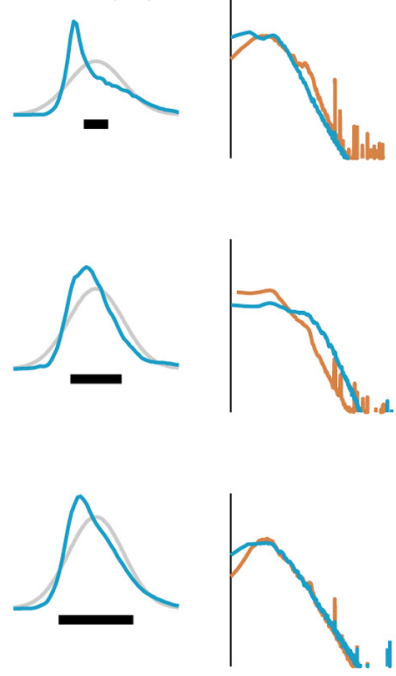

$\left.\right|_{0} ^{1}$
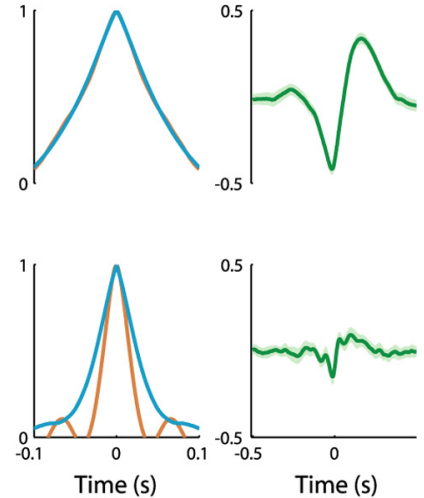

Figure 1. $V_{\mathrm{m}}$ and LFP recordings in rat $\mathrm{S1}$ exhibit a rich repertoire of network dynamics. $A$, Examples of $V_{\mathrm{m}}$ and nearby LFP simultaneous recordings in 5 different neurons. Spikes are clipped. $\boldsymbol{B}$, The $V_{\mathrm{m}}$ distributions. For comparison, a normal distribution having the same SD is shown. The scale bar below each distribution is $5 \mathrm{mV}$. The $V_{\mathrm{m}}$ distributions vary from bimodal, as in neuron $\mathbf{1}$, to normal, as in neuron 5. C, The power and autocorrelation of the $V_{\mathrm{m}}$ and the LFP signals, normalized to have a unit SD. D, Cross-correlation between $V_{\mathrm{m}}$ and LFP. Shaded region shows the $95 \%$ confidence limits. (The examples presented in each of the Figs. 1-4, and 8 are from distinct neurons.)

Sharp recordings which did not pass the criteria of baseline voltage below $-50 \mathrm{mV}$, and spikes that are at least $50 \mathrm{mV}$ high were not included in the recordings database. Although we were able to obtain stable sharp recordings of several minutes, which is well beyond the results reported by Fee (2000) for recordings without active mechanical stabilization, typically we could not obtain recordings of tens of minutes, as reported in the work of Timofeev et al. (2001) in head-fixed cats. The stability of the whole-cell recordings was better. All the recordings included in the analysis were performed without any current injection via the electrode $\left(I_{\text {inj }}=0\right)$.

The recordings exhibited a rich repertoire of network dynamics (Figs. $1 A-C, 2 A-C$ ), ranging from Up-Down and other slow activities as in the work of Poulet and Petersen (2008), which are common to the quiet wakefulness states, and up to fast asynchronous activity, reminiscent of the high conductance states described by Rudolph et al. (2007). In 37 of the 41 recorded neurons, the $V_{\mathrm{m}}$ and LFP signals were significantly correlated (S1, Fig. $1 D$; PFC, Fig. $2 D$ ). The marked differences in $V_{\mathrm{m}}$ and LFP dynamics (Figs. $1 A-C, 2 A-C$ ) and in the cross-correlation between $V_{\mathrm{m}}$ and LFP (VmLFPcc, Figs. 1D, 2D) across the experiments are to a large extent due to the distinct behavioral states of the animal at the time of the recording (also see below).

\section{VmLFPcc and LFP STA have similar waveform}

To reveal how the correlation between $V_{\mathrm{m}}$ and LFP is related to the correlation between the spikes of the neuron and the LFP, we have compared the waveforms of VmLFPcc and LFP STA. For the analysis, we have pooled together the $\mathrm{S} 1$ and the PFC recordings. From the 37 neurons whose membrane potential exhibited a significant correlation with the LFP, 11 neurons were not used because of a very low firing rate, which precluded collecting at least 30 spikes (e.g., the neurons presented in Fig. 1 A1, A4). The spiking of the neurons used in the analysis was irregular, the coefficient of variation $(\mathrm{CV})$ of the interspike intervals (ISIs) was $1.19 \pm 0.76(n=26)$, in agreement with what is known from extracellular recordings (Softky and Koch, 1993; Shadlen and Newsome, 1998; de Kock et al., 2007; Averbeck, 2009).

We found a remarkable similarity between the shape of the LFP STA and the shape of the VmLFPcc over a large range of firing rates and for cells exhibiting different repertoire of activities. Figure 3, $A-D$, presents examples of recordings from four neurons, with LFP STA superimposed with the VmLFPcc, by normalizing the former to match the norm of the crosscorrelation (see Materials and Methods). The distribution of the similarity score for all the analyzed neurons (Fig. $3 E$ ) indicates that the two waveforms resemble each other. 
A

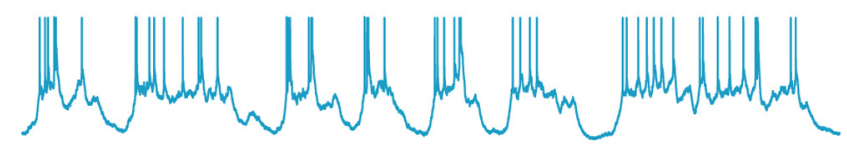

1

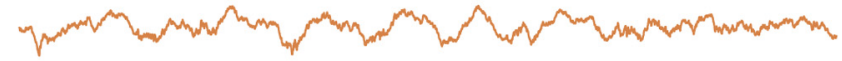

2

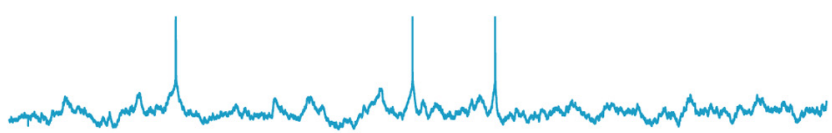

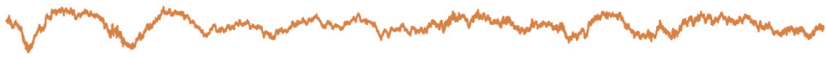

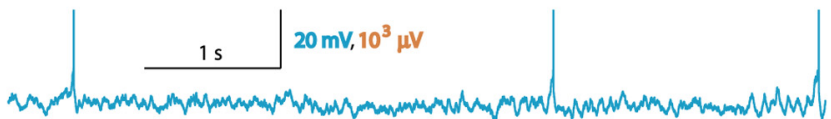

3

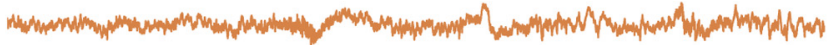

B
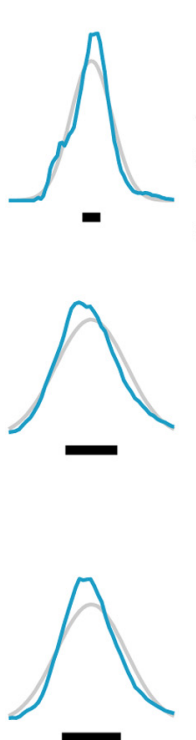

C
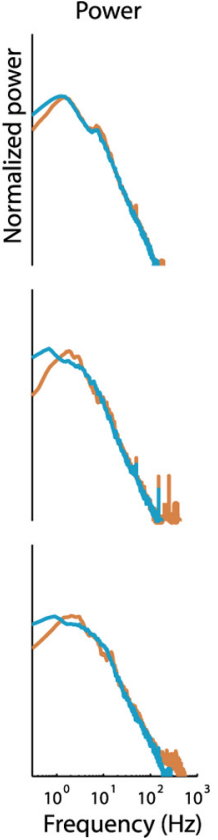

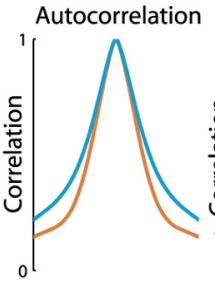

VmLPFCC
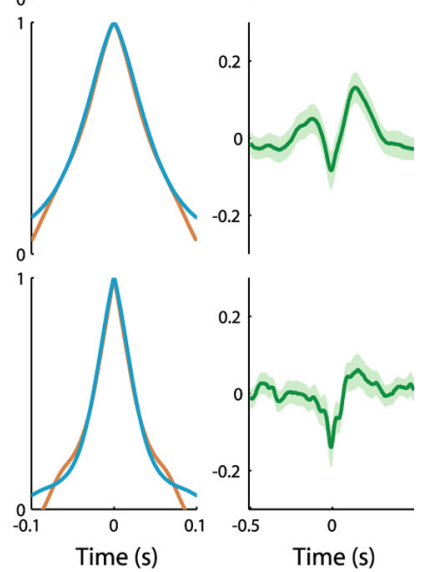

Figure 2. $\quad V_{m}$ and LFP recordings in rat PFC exhibit a rich repertoire of network dynamics. The figure is in the same format as Figure 1, presenting 3 examples of simultaneous $V_{m}$ and nearby LFP recordings performed in the PFC.

A

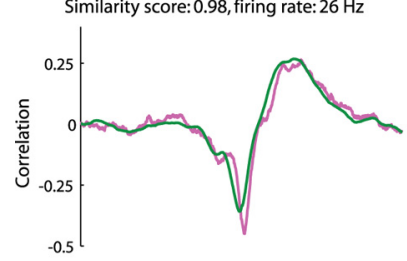

Cimilarity score: 0.76 , firing rate: $12 \mathrm{~Hz}$

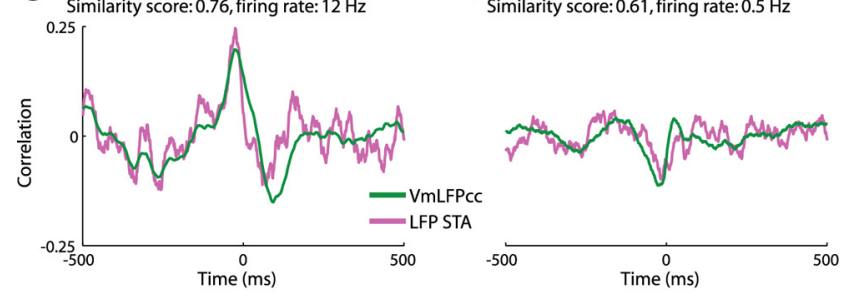

E

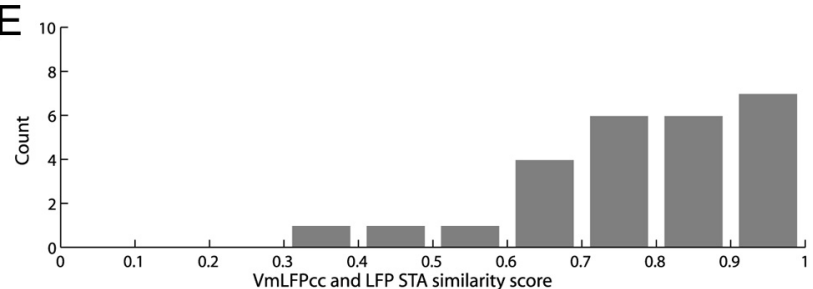

Figure 3. In awake rats LFP STA and VmLFPcc have similar waveform. $A-D$, Examples of VmLFPcc and scaled LFP STAs in 4 neurons. The neuron in $B$ was recorded in PFC, the rest in S1. $\boldsymbol{E}$, Distribution of the similarity scores (see Materials and Methods) between LFP STA and VmLFPcc in all the analyzed neurons $(n=26)$.

The VmLFPcc and LFP STA waveforms are mainly determined by the low-frequency component of the LFP and $V_{\mathrm{m}}$, since most of the power is concentrated in this band (Figs. $1 C, 2 C$ ). The similarity of VmLFPcc and LFP STA however can be observed in higher frequencies as well, by filtering out the low-frequency
A<smiles>[R10][R11]([H])([H])[H]</smiles>

Raw LFP and Vm

B

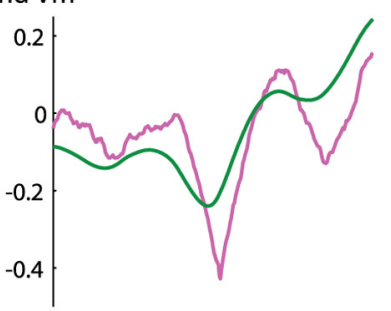

LFP and Vm high-pass filtered above $25 \mathrm{~Hz}$
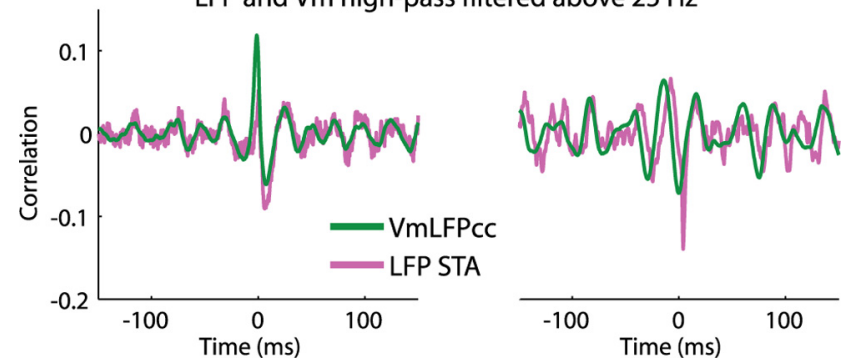

Figure 4. VmLFPcc and LFP STA waveform similarity extends to gamma frequencies. $A$, Examples of VmLFPcc and scaled LFP STA in $S 1$ and PFC neurons (as in Fig. $3 A-D$ ). The firing rates of the neurons were 12 and $8 \mathrm{~Hz}$, respectively. $B, V m L F P c c$ and LFP STA for the same two neurons, computed after the signals were digitally (offline) high-pass filtered $>25 \mathrm{~Hz}$.

component. Two recordings, exhibiting significant gamma frequency $V_{\mathrm{m}}$-LFP and spike-LFP correlation, revealed by high-pass filtering of the two signals $>25 \mathrm{~Hz}$, are presented in Figure 4 . Even if the signals are correlated in a large range of high frequencies, because of the $1 / \mathrm{f}$ distribution of the power in the gamma band (Figs. $1 C, 2 C$ ), the two waveforms mainly exhibit similar oscillatory activity in the lowest frequencies which were not filtered out. The more appropriate way for examining the relationships between $V_{\mathrm{m}}$, spikes and LFP in all the frequency bands, regardless of their power, is to use coherency analysis. This data will be presented later on. 
A priori, the similarity between VmLFPcc and LFP STA can be explained in two different ways. It might be the case that the VmLFPcc is mainly determined by the activity in the short intervals containing the spikes, while in the intervals in between the spikes the correlation is low or averaged out. For example, this is expected to be the case if spikes are driven by synaptic excursions much larger than the typical subthreshold activity, especially if these excursions are due to an input shared by the whole local network. On the other hand, it is possible that the VmLFPcc is determined by the entire time course of the two signals, and LFP STA simply reflects this correlation. To address these possibilities, we have compared the crosscorrelation of $V_{\mathrm{m}}$ and LFP with their cross-correlation where an interval around each spike was not taken into account (see Materials and Methods). We found that the cross-correlation waveform remains essentially the same even after activity intervals of $40-200 \mathrm{~ms}$ around each spike were excluded (Fig. 5A-C). In addition, we have compared the $V_{\mathrm{m}}$-LFP coherence of the entire traces with their coherence in windows without spikes only (here $0.2 \mathrm{~s}$ windows and 3-taper estimates were used). The latter was found to be similar or even higher than the former in all the frequencies up to $100 \mathrm{~Hz}$ (Fig. 5D). Both results indicate that the shape of VmLFPcc is determined neither by the spikes (which were digitally removed from the $V_{\mathrm{m}}$ traces), nor by the intervals right around them, but by the entire time course of the two signals. Thus, in our recordings LFP STA reflects the pervasive synaptic correlations between the cell and the population, rather than being shaped by specific, rapid network events.

In contrast to the similar waveform of VmLFPcc and LFP STA typically observed in awake animals, in recordings under deep halothane anesthesia an STA with very sharp negative peak around $0 \mathrm{lag}$, reflecting the LFP component locked to the spikes with a precision of a few milliseconds, was common (Fig. 6). Whereas in awake recordings the ratio of peak magnitudes of LFP STA and VmLFPcc, after both were normalized to have the same norm (see Materials and Methods), was $1.3 \pm 0.2$, in the anesthetized recordings presented in Figure 6 this ratio was $>2$.

\section{VmLFPcc and LFP STA are correlated in magnitude}

Next, we examined how the magnitude of the LFP STA is related to the magnitude of VmLFPcc. In our experiments, the SD of the LFP signal, and the amplitude of LFP STA were in the 2-200 $\mu \mathrm{V}$ range, compatible with previous studies (Eggermont and Smith, 1995; Fries et al., 2001; Pesaran et al., 2002; Goldberg et al., 2004; Poulet and Petersen, 2008; Ray et al., 2008; Nauhaus et al., 2009). First, we have compared the absolute peak amplitudes of LFP STA and of VmLFPcc, multiplied by the SD of the LFP signal. The scaled VmLFPcc is the most straightforward analog of LFP STA, as it is equal to the cross-covariance of a $z$-scored $V_{\mathrm{m}}$ and the LFP, normalized by the length of the recording. As shown in Figure $7 \mathrm{~A}$, the two measures are highly correlated. This correlation, however, is partly due to the variability in the LFP magnitude across the experiments, incorporated in both measures. Hence, we also compared the correlation between the magnitudes of LFP STA, normalized (divided) by the SD of LFP, and VmLFPcc (Fig. 7B). As expected, this correlation is lower, but still highly significant. The correlations presented in Figure 7, $A$ and $B$, also hold separately for the magnitudes of the negative ( $p<0.001$ and $p=0.001$, respectively) and the positive peaks ( $p<0.001$ and $p=0.034$, respectively).

In addition, we have examined the relationship between the $V_{\mathrm{m}}$-LFP and spike-LFP coherences, which are synchrony measures independent of the $V_{\mathrm{m}}$ and LFP power in different frequency bands. As expected, in the low frequencies, higher $V_{\mathrm{m}}$-LFP coherence implies higher spike-LFP coherence and vice versa (Fig. $7 C$ ). In the gamma band, 14 of the 26 neurons displayed a significant (see Materials and Methods) $V_{\mathrm{m}}$-LFP coherence. Of these, 8 also had significant spike-LFP coherence of a highly correlated magnitude (Fig. 7D; just one neuron had significant spike-LFP but not $V_{\mathrm{m}}$-LFP coherence). Importantly, a much smaller coherence was found $>25 \mathrm{~Hz}$, indicating that $V_{\mathrm{m}}$ and spikes are not precisely locked to the LFP at these high frequencies.

Several considerations suggest that the variation in the distance between the tips of the intracellular and the LFP electrodes across the experiments does not contribute substantially to the magnitude correlation between the VmLFPcc and normalized LFP STA, and between $V_{\mathrm{m}}$-LFP and spike-LFP coherences. First, the variability in the magnitudes seems to be too high to be ex- 


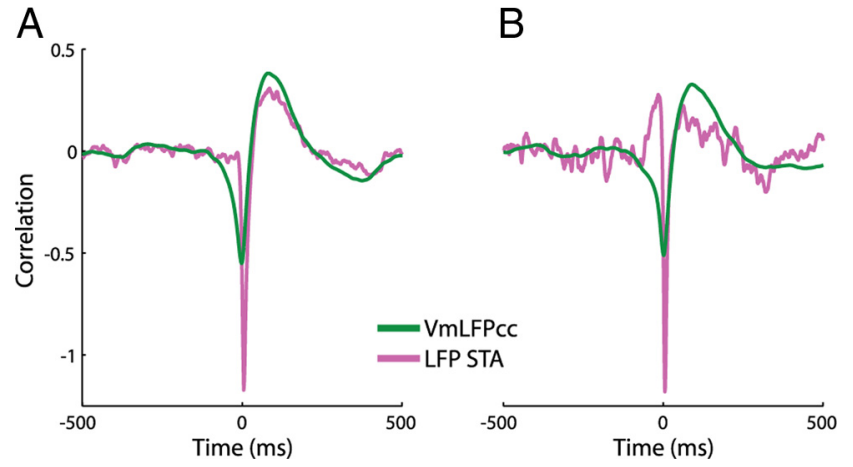

Figure 6. Under deep anesthesia precise spike-LFP locking is common. Examples showing that the waveforms of VmLFPcc and LFP STA in animals under deep halothane anesthesia are distinct. These two cells (which are shown only here and are not part of any other analysis) exhibit the case where a large portion of the spikes has a tight temporal relation with the LFP signal. The firing rates of the neurons were $\sim 6 \mathrm{~Hz}(\boldsymbol{A})$ and $\sim 1 \mathrm{~Hz}(\boldsymbol{B})$. The ratios between absolute peaks of LFP STA and VmLPFcc in the figure (after both were normalized to have the same Euclidian norm) are 2.1 and 2.3, respectively.

plained by the distance factor, which we tried to preserve across the experiments. Furthermore, the magnitude of VmLFPcc was correlated with the magnitude of the LFP signal, which is determined by the cortical dynamics and is independent of the distance (supplemental Fig. 1, available at www.jneurosci.org as supplemental material). Finally, a similar dependence was observed across network state transitions during recordings of single neurons, as we show next. We therefore conclude that the strength of $V_{\mathrm{m}}$ and LFP correlation, which reflects the correlation of synaptic inputs to individual neuron and the entire network, can be predicted in a relatively accurate manner from the LFP STA.

\section{Shape similarity and magnitude correlation are retained during state transitions}

Occasionally during the recordings the behavior of the animal switched between different states, typically from some form of quiet state to grooming or licking juice, or vice versa. The state transitions were accompanied by a substantial change in the dynamics of the brain activity, evident in both the $V_{\mathrm{m}}$ and the LFP recordings (Fig. $8 A, B$; supplemental Movies 1 and 2, available at www.jneurosci.org as supplemental material). In Figure $8 A$ the transition is from slow activity, characterized by large fluctuations of membrane potential and LFP, to rapid activity. Figure $8 B$ presents a transition from rapid to slow activity. These transitions appear to have a similar nature to transitions reported to occur in the barrel cortex of awake mice during whisking (Poulet and Petersen, 2008), although here they were not related to whisking per se, and in particular were observed in the PFC recordings as well. The marked changes in VmLFPcc caused by the state transition were accompanied by similar changes in both the waveform and the amplitude of LFP STA. In the first example, VmLFPcc was substantially higher before the transition and so was the magnitude of the LFP STA (Fig. $8 C$ ). In the second example opposite change in magnitude was observed (Fig. 8D). In both neurons and in each state the shape of VmLFPcc and the corresponding LFP STA were remarkably similar (Fig. 8C,D). These data indicate again that the latter measure can predict changes in synaptic correlation in the network (Fig. 8C-E). Importantly, in some transitions the change in LFP STA magnitude covered almost the entire population range and was accompanied by a corresponding change in the VmLFPcc
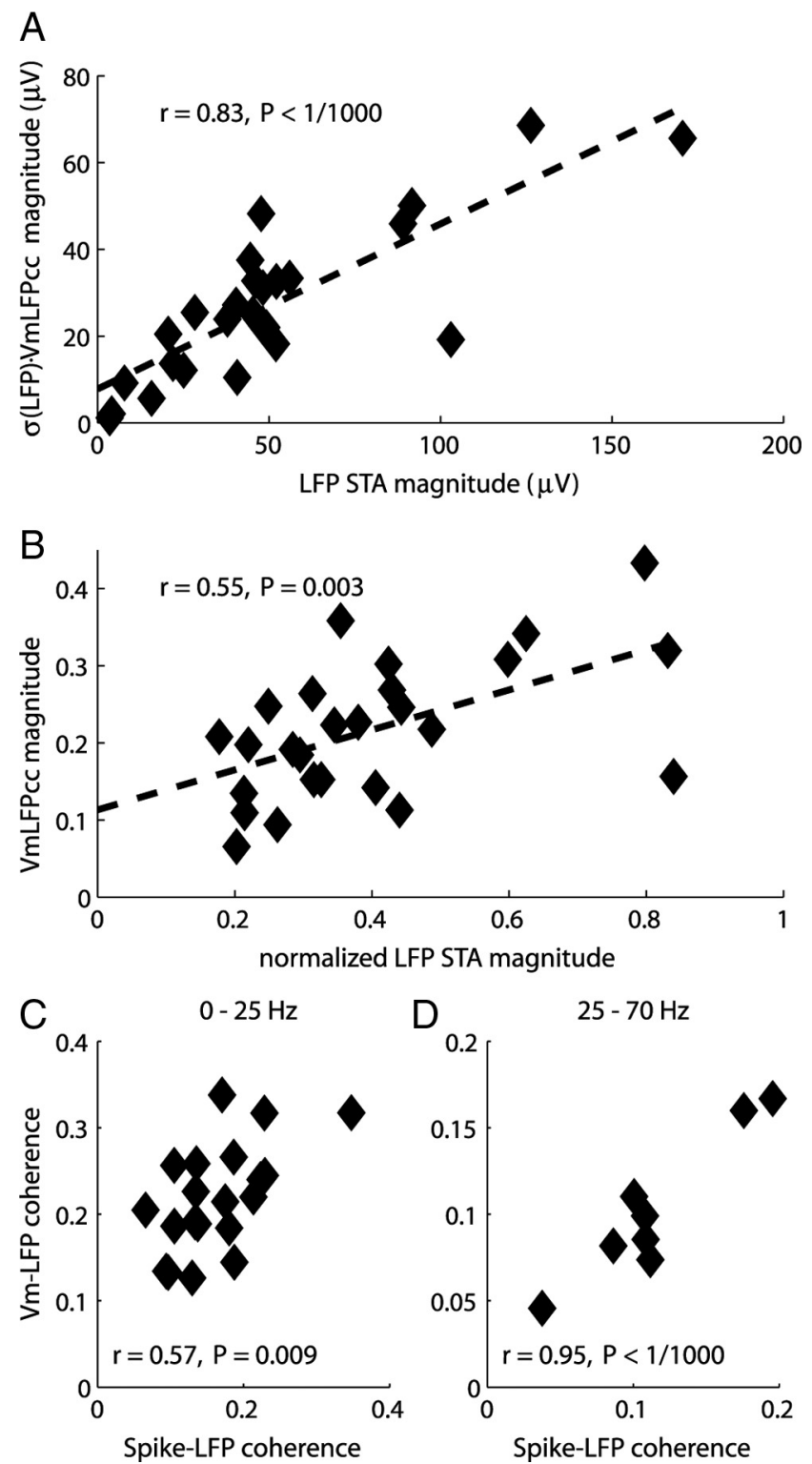

Figure 7. LFP STA and VmLFPcc are correlated in magnitude. $\boldsymbol{A}$, The magnitudes of VmLPFcc scaled by LFP SD and of LFP STA, both quantified by the value of their absolute peak, are significantly correlated. The dashed line shows the linear regression ( $y=0.38 x+7.8$ ). $\boldsymbol{B}$, The magnitudes of VmLPFcc and of normalized LFP STA, quantified by the value of their absolute peak, are significantly correlated. The dashed line shows the linear regression ( $y=0.26 x+$ 0.11). The reduction in the correlation between the two measures (compared to $A$ ) is expected, as in $\boldsymbol{A}$ both coordinates are multiplied by LFPSD. C, D, The $V_{\mathrm{m}}$-LFP and spike-LFP coherences are correlated, both in the low $(\boldsymbol{C})$ and gamma $(\boldsymbol{D})$ frequency bands. Only neurons in which both the $V_{\mathrm{m}}$-LFP and spike-LFP coherences were significant in more than half of the frequency band (see Materials and Methods) were included. Including all the neurons would have further increased the correlation.

(Fig. $9 A, B$ ). Similarly, a comparison of the $V_{\mathrm{m}}$-LFP and spikeLFP coherences in the $0-25 \mathrm{~Hz}$ frequency band showed that during faster activity both measures change in a compatible way (Fig. 9C).

To summarize, the analysis of state transitions confirms that the conclusions regarding the shape similarity and amplitude correlation between LFP STA and VmLFPcc, reached based on a set of recordings from different neurons, also hold for the very same neuron while the network switches between different types of activity. 


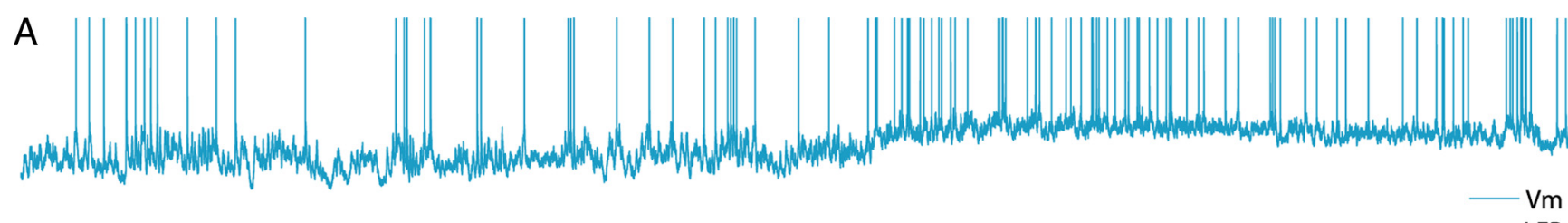

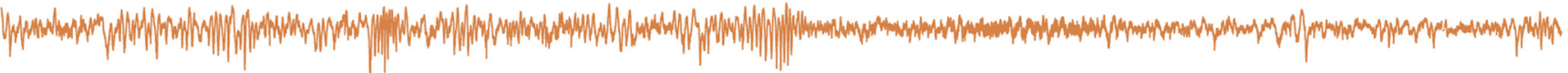

B $3 \mathrm{~s} \quad 10 \mathrm{mV}, 10^{3} \mu \mathrm{V}$

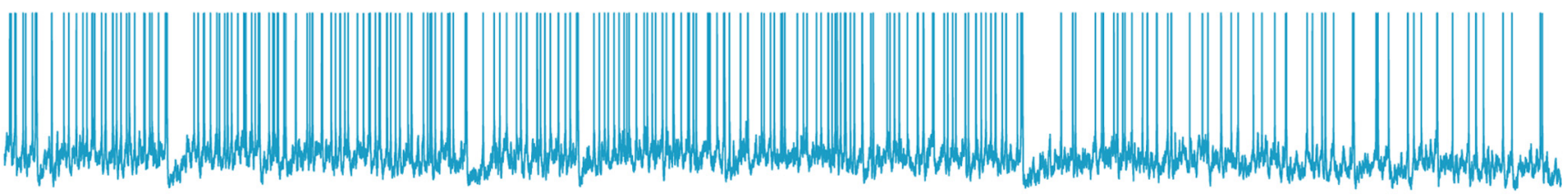

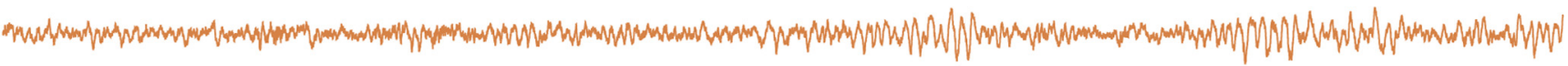
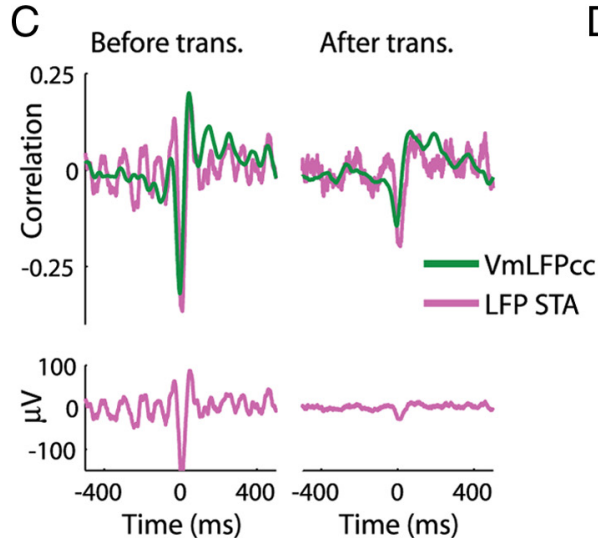

Before trans.

After trans.
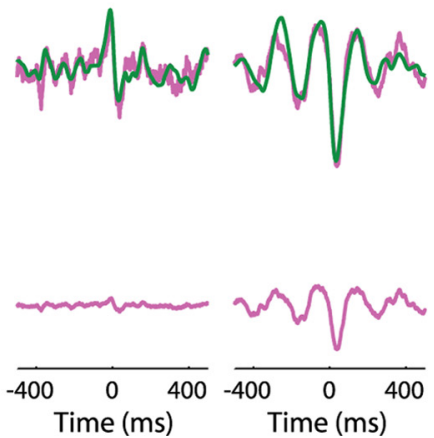

E

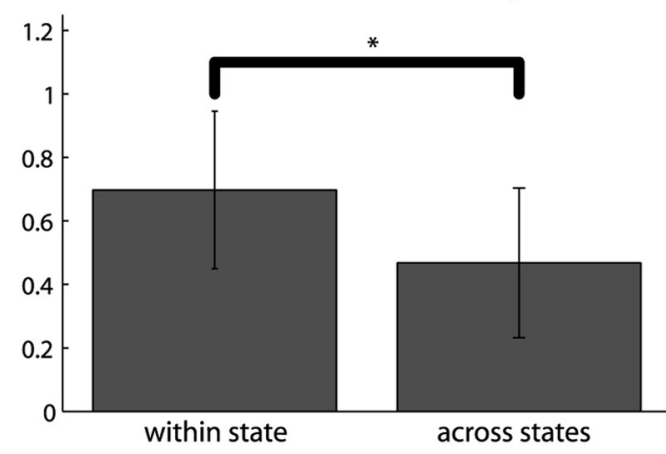

Figure 8. Shape correlations between LFP STA and VmLFPcc are preserved during transitions between different states of cortical activity. $A, B$, Examples of simultaneous $V_{m}$ and nearby LFP recordings in $S 1$ during state transitions. In $\boldsymbol{A}$, the transition is from a slow to fast activity, these transitions are typically abrupt. In $\boldsymbol{B}$, the transition is from fast to slow activity, such transitions are usually more gradual. The bars below indicate the transition direction, from slow (white) to fast (black) or vice versa. The firing rates of the two neurons before and after the transition were 2.4, 5.7 , and $15.4,5.9 \mathrm{~Hz}$, respectively. $\boldsymbol{C}, \boldsymbol{D}, V \mathrm{mLFPcc}$ and scaled LFP STA for the two neurons in $\boldsymbol{A}, \boldsymbol{B}$, before and after the transition. The LFP STA is shown again below in $\mu V$. Data were computed from (50, 35 ) seconds before and $(84,39)$ seconds after the transitions, respectively. $\boldsymbol{E}$, The VmLFPcc waveform is more similar to the LFP STA waveform in the same state than to the LFP STA in a different state (data from 13 state transitions, $p=0.002$, nonpaired $t$ test).

\section{Discussion}

In the present work, we investigated how the spike-triggered average of a nearby LFP signal (LFP STA) is related to the cross-correlation of the neuron's membrane potential with the LFP (VmLFPcc). Both measures are second-order (i.e., linear) statistics which correlate the activity of the neuron with the LFP signal. From experimental viewpoint, LFP STA is readily available from extracellular recordings and thus is frequently used. In contrast, VmLFPcc requires intracellular recordings, which are more technically demanding to obtain, especially in awake animals (as in the present study). Understanding the relationship between the two might be useful for interpreting data from extracellular recording experiments where intracellular recordings are very hard or
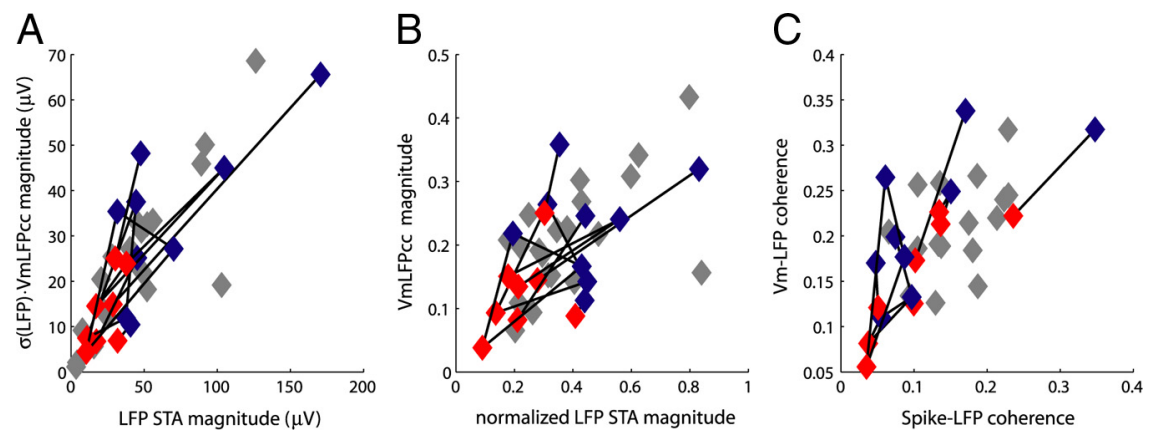

Figure 9. Magnitude correlations between LFP STA and VmLFPcc are preserved during transitions between different states of cortical activity. The magnitudes of VmLFPcc and LFP STA vary together during state transitions. The connected blue and red points represent the slower and the faster activities recorded in the same neuron, respectively. $\boldsymbol{A}$, The magnitudes of $V m L P F c c$ scaled by LFP SD and of LFP STA. For comparison, the points from Figure $7 A$ are shown in the background (gray). Note that the transitions cover a large range of the population. $\boldsymbol{B}$, The magnitudes of VmLPFcc and of normalized LFP STA. For comparison, the points from Figure $7 B$ are shown in the background (gray). $C$, The $V_{\mathrm{m}}$-LFP coherence and spike-LFP coherence in the $0-25 \mathrm{~Hz}$ band. For comparison, the points from Figure 7 ( are shown in the background (gray). 
even impossible to obtain, such as in human and primate studies (Fries et al., 2001; Pesaran et al., 2002; Nir et al., 2007) or when a large number of sites are recorded with multielectrode arrays (Nauhaus et al., 2009).

The correlation of spike trains and LFP is commonly used as a measure of synchrony between the single neurons and the mean synaptic currents in the nearby population, in particular in case of oscillatory activity. In the primary visual cortex, correlated gamma frequency oscillations of spikes and LFP in response to stimulus presentation are a well known example for such a synchronous activity (Singer and Gray, 1995). In many works, the relation between spike trains and LFP was used to elucidate how changes in specific experimental conditions of interest affect synchronization in local cortical circuits. In more recent studies, the correlation between the spikes and the LFP was investigated using frequency domain methods in addition to the time domain ones (Kohn et al., 2009). Examples of specific conditions in which LFP STA was used to look for significant difference in network synchrony include waking states (Destexhe et al., 1999), artificially induced Parkinson's syndrome (Goldberg et al., 2004), visual and auditory stimuli (Eggermont and Smith, 1995; Henrie and Shapley, 2005; Nauhaus et al., 2009), attention to visual stimuli (Fries et al., 2001) and activation of working memory (Pesaran et al., 2002), to name just a few.

Our observations of the similarity between the waveforms of VmLFPcc and LFP STA (Fig. 3) provide direct evidence for the commonly held view that the correlation between spikes and LFP reflects synchrony between the synaptic inputs, which drive the membrane potential fluctuations of the neuron, and the synaptic activity in the local network. Furthermore, we found a quantitative correspondence between the magnitude of the spike-LFP correlation and the VmLFPcc (Fig. 7), which allows to make gross estimates of the synaptic correlation between the population activity and individual neurons from (existing) extracellular recordings data. The observed magnitude correlation is not a result of the variability in the distance between the recording sites of the cell and the LFP across the experiments, as shown in the Results section above. Although here the magnitude correspondence was established only for a range of spontaneous activities and mainly for the primary somatosensory cortical area (S1), it probably applies in a more general case. It is important to note, however, that there is no fine, one-to-one correspondence between the two measures, as was revealed by our analysis of state transitions, in which two distinct networks states, producing different LFP STA and VmLFPcc, were observed via the same neuron and LFP recording site. State transitions confirmed that on the crude scale higher LFP STA goes hand in hand with stronger $V_{\mathrm{m}}$-LFP correlation, however we also observed several transitions in which small changes in the opposite directions occurred (e.g., VmLFPcc decreased while normalized LFP STA increased; see Fig. 9B). Whether similarity between LFP STA and VmLFPcc exists in conditions other than spontaneous activity remains to be explored.

The correspondence between VmLFPcc and LFP STA might not hold for sparsely firing neurons (firing rate on the order of $0.01 \mathrm{~Hz}$ and less), several of which we have encountered (Fig. $1 A 1, A 4)$. In these cells spikes ride on top of synaptic bumps that seem rather distinct from the main synaptic activity. Unfortunately, the recording durations did not allow collecting a sufficient number of spikes for LFP STA analysis in these neurons.

The synchrony observed here between the $V_{\mathrm{m}}$ and the LFP in awake animals was mainly due to activity in the low frequencies. In addition to the higher power in these frequencies (Figs. 1C,
$2 C)$, the average coherence in the $0-25 \mathrm{~Hz}$ band was $0.21 \pm 0.06$, whereas in the $25-70 \mathrm{~Hz}$ gamma band it was only $0.08 \pm 0.04$ (and significant only in 14 of 26 neurons), consistently with results from dual intracellular recordings in the barrel cortex of awake mice (Poulet and Petersen, 2008). Furthermore, VmLFPcc and $V_{\mathrm{m}}$-LFP coherence were almost unaffected when intervals of up to $200 \mathrm{~ms}$ around spikes were not taken into account (Fig. 5), suggesting that occurrences of spikes are not related to specific changes in the coherence of the signals. In some cases we even observed an increase in $V_{\mathrm{m}}$-LFP coherence in periods without spikes, when compared with their coherence over the entire time course (Fig. 5D), suggesting that in these cases the synchrony between the network activity and the synaptic inputs which elicited the spikes was even lower than the average one.

The above observations agree with the widely held view that the slower fluctuations in the cortex modulate the faster events, which together with the intrinsic properties precisely control the spike times (Buzsaki, 2006; Tiesinga et al., 2008). Highly precise firing probably occurs in small groups of neurons, hence might not be prominent in the LFP signal, which represents an average over a population of several thousand neurons. In the study by Poulet and Petersen (2008), the spike-triggered average of the membrane potential in the nearby cell showed a very small depolarization $(\sim 1.5 \mathrm{mV}$ compared with $\sim 9 \mathrm{mV}$ in the spiking neuron itself), providing another compelling evidence that spiking during spontaneous activity in the primary somatosensory cortex of awake rodents is not elicited by specific strong and rapid events that coexist in the population activity and the neuron.

The VmLFPcc and LFP STA are two distinct measures of correlation between the population and the single neuron activities, the former based on the neuron's synaptic input and the latter on its spike output. The similarity between VmLFPcc and LFP STA, observed in the awake recordings, suggests that the $V_{\mathrm{m}}$-to-spike transformation 'computed' by the neuron is in large part averaged out in the temporal and spatial mean expressed by these two measures. The differences that do exist between the waveforms of LFP STA and VmLFPcc, especially in the deeply anesthetized condition (Fig. 6), might provide important information about the transformation from $V_{\mathrm{m}}$ to spikes, and warrant a further investigation.

In summary, our findings indicate that for a large range of firing rates and for different cortical states LFP STA in awake rats provides an estimation of both the time course and the magnitude of $V_{\mathrm{m}}$-LFP cross-correlation.

\section{References}

Averbeck BB (2009) Poisson or not Poisson: differences in spike train statistics between parietal cortical areas. Neuron 62:310-311.

Buzsaki G (2006) Rhythms of the brain. New York: Oxford UP.

de Kock CP, Bruno RM, Spors H, Sakmann B (2007) Layer- and cell-typespecific suprathreshold stimulus representation in rat primary somatosensory cortex. J Physiol 581:139-154.

Destexhe A, Contreras D, Steriade M (1999) Spatiotemporal analysis of local field potentials and unit discharges in cat cerebral cortex during natural wake and sleep states. J Neurosci 19:4595-4608.

Deweese MR, Zador AM (2004) Shared and private variability in the auditory cortex. J Neurophysiol 92:1840-1855.

Eggermont JJ, Smith GM (1995) Synchrony between single-unit activity and local field potentials in relation to periodicity coding in primary auditory cortex. J Neurophysiol 73:227-245.

Fee MS (2000) Active stabilization of electrodes for intracellular recording in awake behaving animals. Neuron 27:461-468.

Fries P, Reynolds JH, Rorie AE, Desimone R (2001) Modulation of oscillatory neuronal synchronization by selective visual attention. Science 291:1560-1563.

Goldberg JA, Rokni U, Boraud T, Vaadia E, Bergman H (2004) Spike syn- 
chronization in the cortex/basal-ganglia networks of Parkinsonian primates reflects global dynamics of the local field potentials. J Neurosci 24:6003-6010.

Gray CM, Singer W (1989) Stimulus-specific neuronal oscillations in orientation columns of cat visual cortex. Proc Natl Acad Sci U S A 86:16981702.

Haider B, Duque A, Hasenstaub AR, McCormick DA (2006) Neocortical network activity in vivo is generated through a dynamic balance of excitation and inhibition. J Neurosci 26:4535-4545.

Hasenstaub A, Shu Y, Haider B, Kraushaar U, Duque A, McCormick DA (2005) Inhibitory postsynaptic potentials carry synchronized frequency information in active cortical networks. Neuron 47:423-435.

Heiss JE, Katz Y, Ganmor E, Lampl I (2008) Shift in the balance between excitation and inhibition during sensory adaptation of S1 neurons. J Neurosci 28:13320-13330.

Henrie JA, Shapley R (2005) LFP power spectra in V1 cortex: the graded effect of stimulus contrast. J Neurophysiol 94:479-490.

Katz Y, Heiss JE, Lampl I (2006) Cross-whisker adaptation of neurons in the rat barrel cortex. J Neurosci 26:13363-13372.

Katzner S, Nauhaus I, Benucci A, Bonin V, Ringach DL, Carandini M (2009) Local origin of field potentials in visual cortex. Neuron 61:35-41.

Kohn A, Zandvakili A, Smith MA (2009) Correlations and brain states: from electrophysiology to functional imaging. Curr Opin Neurobiol 19:434438.

Lampl I, Reichova I, Ferster D (1999) Synchronous membrane potential fluctuations in neurons of the cat visual cortex. Neuron 22:361-374.

Logothetis NK (2008) What we can do and what we cannot do with fMRI. Nature 453:869-878.

Mehring C, Rickert J, Vaadia E, Cardosa de Oliveira S, Aertsen A, Rotter S (2003) Inference of hand movements from local field potentials in monkey motor cortex. Nat Neurosci 6:1253-1254.

Mitra PP, Bokil H (2008) Observed brain dynamics. New York: Oxford UP.

Mitzdorf U (1985) Current source-density method and application in cat cerebral-cortex-investigation of evoked-potentials and EEG phenomena. Physiol Rev 65:37-100.

Nauhaus I, Busse L, Carandini M, Ringach DL (2009) Stimulus contrast modulates functional connectivity in visual cortex. Nat Neurosci 12: $70-76$.

Niessing J, Ebisch B, Schmidt KE, Niessing M, Singer W, Galuske RA (2005) Hemodynamic signals correlate tightly with synchronized gamma oscillations. Science 309:948-951.

Nir Y, Fisch L, Mukamel R, Gelbard-Sagiv H, Arieli A, Fried I, Malach R (2007) Coupling between neuronal firing rate, gamma LFP, and BOLD fMRI is related to interneuronal correlations. Curr Biol 17:1275-1285.
Okun M, Lampl I (2008) Instantaneous correlation of excitation and inhibition during ongoing and sensory-evoked activities. Nat Neurosci 11:535-537.

Pesaran B (2009) Uncovering the mysterious origins of local field potentials. Neuron 61:1-2.

Pesaran B, Pezaris JS, Sahani M, Mitra PP, Andersen RA (2002) Temporal structure in neuronal activity during working memory in macaque parietal cortex. Nat Neurosci 5:805-811.

Poulet JF, Petersen CC (2008) Internal brain state regulates membrane potential synchrony in barrel cortex of behaving mice. Nature 454:881-885.

Rasch MJ, Gretton A, Murayama Y, Maass W, Logothetis NK (2008) Inferring spike trains from local field potentials. J Neurophysiol 99:1461-1476.

Ray S, Hsiao SS, Crone NE, Franaszczuk PJ, Niebur E (2008) Effect of stimulus intensity on the spike-local field potential relationship in the secondary somatosensory cortex. J Neurosci 28:7334-7343.

Rudolph M, Pospischil M, Timofeev I, Destexhe A (2007) Inhibition determines membrane potential dynamics and controls action potential generation in awake and sleeping cat cortex. J Neurosci 27:5280-5290.

Shadlen MN, Newsome WT (1998) The variable discharge of cortical neurons: implications for connectivity, computation, and information coding. J Neurosci 18:3870-3896.

Singer W, Gray CM (1995) Visual feature integration and the temporal correlation hypothesis. Annu Rev Neurosci 18:555-586.

Softky WR, Koch C (1993) The highly irregular firing of cortical cells is inconsistent with temporal integration of random EPSPs. J Neurosci 13:334-350.

Steriade M (2001) Impact of network activities on neuronal properties in corticothalamic systems. J Neurophysiol 86:1-39.

Steriade M, Amzica F, Contreras D (1996) Synchronization of fast (30-40 $\mathrm{Hz}$ ) spontaneous cortical rhythms during brain activation. J Neurosci 16:392-417.

Tiesinga P, Fellous JM, Sejnowski TJ (2008) Regulation of spike timing in visual cortical circuits. Nat Rev Neurosci 9:97-107.

Timofeev I, Grenier F, Steriade M (2001) Disfacilitation and active inhibition in the neocortex during the natural sleep-wake cycle: an intracellular study. Proc Natl Acad Sci U S A 98:1924-1929.

Viswanathan A, Freeman RD (2007) Neurometabolic coupling in cerebral cortex reflects synaptic more than spiking activity. Nat Neurosci 10:1308-1312.

Volgushev M, Chauvette S, Mukovski M, Timofeev I (2006) Precise longrange synchronization of activity and silence in neocortical neurons during slow-wave oscillations [corrected]. J Neurosci 26:5665-5672.

Xing D, Yeh CI, Shapley RM (2009) Spatial spread of the local field potential and its laminar variation in visual cortex. J Neurosci 29:11540-11549. 